\title{
Performance Evaluation of Signal and Image Processing Systems
}

\author{
Firooz A. Sadjadi, MEMBER SPIE \\ Honeywell, Inc. \\ Systems and Research Center \\ 3660 Technology Drive \\ Minneapolis, Minnesota 55418
}

The development of signal and image processing algorithms and systems during the past few decades has been phenomenal. This rapid progress has led to a need to assess what has been developed and to communicate this assessment to the signal and image processing community. It is this need that motivated me to initiate the First International Conference on Signal and Image Processing Performance Evaluation in 1990 and this Special Section of Optical Engineering on this topic.

The performance evaluation problem refers to finding where and how well the algorithms and systems work, where and why they perform poorly, and what exactly they accomplish. The performance evaluation field has come a long way. Like many new fields of science and technology, it has grown out of necessity from other fields. That necessity has been the building of working and reliable signal and image processing systems. People who are a bit removed from the practical aspects of the image and signal processing field have had the attitude of ignoring the evaluation issues altogether. Multitudes of algorithms are created every year in academe and the results and claims are usually shown on only a few frames of data. Those who are more involved in the application aspects of the signal processing field have generally been more aware of the evaluation problem. However, they usually had the tendency to view the problem provincially: they created their own definitions of performance measures, test procedures, data requirements, etc. Evaluation was more an art than a scientific endeavor. This inadequacy of thinking is no more pronounced in any domain than in the area of automatic object recognition, where evaluation has become a real bottleneck in the development life cycle. Even though a general scientific tool for performance evaluation, namely, experimental design methodology, has been around since the 1920s, its use and appreciation in the image and signal processing field has not been forthcoming until very recently.

Evaluation technology is maturing now. Specialized software and hardware are being developed yearly for addressing the needs of this technological field. Attitudes in the signal processing community are also changing. A growing number of users and developers are appreciating the performance evaluation problem that is present in every signal and image processing algorithm and system development area. The future is bright!

This Special Section is composed of a mix of invited and contributed papers. The paper by Sadjadi and Bazakos provides a survey of performance evaluation technology in the field of automatic target recognition (ATR). The paper by Clark and Velten addresses the important issues of data characterization. A paper on the same topic by Noah et al. arrived too late to be included here but will be published in a future issue. In the next paper, Bennett discusses a knowledge-based technique for evaluation of the segmentation module of ATR. A paper by Walters describing a facility called AUTOSPEC for the end-to-end evaluation of ATR systems also will be published in a future issue.
Modeling and characterizing the signal processing sensors crucial in any end-to-end evaluation study is addressed by three papers. Blackwell and Kalin address the problem of sensor vibration, Reichenbach et al. discuss the characterization of digital image acquisition devices, and Jutamulia et al. describe a tool for dynamic infrared imagery scene simulation. The last two papers, one by Sadjadi et al. and one by Marshall, deal with the use of system performance estimates for dynamic adaptation of the signal processing systems to changing environmental conditions. Marshall discusses an adaptive self-learning filter for a real-time target pointing control system, while Sadjadi et al. describe a system for automatic adaptation of an ATR system to scene and scenario variations.

I would like to express my appreciation to all of the authors and reviewers who made this issue possible. I am thankful to Professor Jack Gaskill and the entire staff of Optical Engineering for their patience and valuable support.

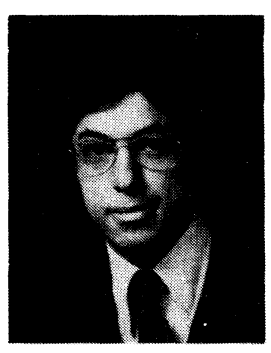

Firooz A. Sadjadi received the BSEE degree from Purdue University in 1972, the MSEE degree in 1974, and the engineer's degree in electrical engineering in 1976 from the University of Southem California. He did postgraduate studies toward the Ph.D. at USC and the University of Tennessee, Knoxville. He worked at the USC Image Processing Institute and at the Image and Pattern Analysis Laboratory of the University of Tennessee and was a consultant to the Oak Ridge National Laboratory. Since 1983 he has been with Honeywell Systems and Research Center, where he is currently a senior research scientist. He specializes in modeling, algorithm design, sensor fusion, and performance evaluation for the development of adaptive millimeter wave radar, infrared, and ladar signal processing systems. He has been the principal investigator and program manager of several automatic target recognition programs for DARPA and other agencies. He is the author of more than 55 technical publications and holds one patent in the area of automatic object recognition and has several others pending. He is the leading author of a forthcoming book, Automatic Target Recognition Systems, to be published by Artech House. He has taught short courses on ATR and multisensor fusion at George Washington University, U.S. government centers, the University of Maryland, UCLA, and at SPIE symposia. He has chaired many conferences and presently is chair of the SPIE conference on Automatic Object Recognition to be held in Orlando, Florida, in April. Mr. Sadjadi is listed in Who's Who in Technology, Who's Who in the Frontiers of Science and Technology, and Who's Who in the World. He is a senior member of IEEE and a member of Sigma Xi and SPIE. He is the recipient of a number of technical awards, among them the Honeywell Technical Achievement Award. 